\title{
I-Xe dating of solar noble gas-rich meteorites
}

\author{
K. ARAI ${ }^{*}$, A. TAKENOUCHI ${ }^{2}$, H. SUMINO ${ }^{1}$ AND \\ S. TACHIBANA ${ }^{3}$
}

${ }^{1}$ Dept. Basic Sci., Univ. Tokyo, Tokyo 153-8902, Japan

(*correspondence: karai@igcl.c.u-tokyo.ac.jp)

${ }^{2}$ Nat. Inst. Polar Res., Tokyo 190-8518, Japan

${ }^{3}$ UTOPS/Dept. Earth Planet. Sci., Univ. Tokyo, Tokyo 1130033, Japan

Brecciated meteorites formed at the surface of planetesimals would have been irradiated by the solar wind (SW) and thus contain abundant SW. In this study, I-Xe ages of Zag (H3-6) and Northwest Africa (NWA) 801 (CR2) breccia meteorites were determined because SW noble gasrich meteorites may potentially constrain the timing of disk gas dissipation in the early Solar System. The SW irradiation onto airless planetesimals occurs only after the disk gas dissipation.

Zag and NWA 801 meteorites were irradiated with neutrons at the Kyoto University research reactor. Xenon isotopes in the samples, including radiogenic ${ }^{129} \mathrm{Xe}$ derived from ${ }^{129} \mathrm{I}$ and ${ }^{128} \mathrm{Xe}$ produced from ${ }^{127} \mathrm{I}$ by $(\mathrm{n}, \beta)$ reaction in the reactor, were released by stepwise heating in vacuum and measured using a noble gas mass spectrometer. The obtained ${ }^{129} \mathrm{Xe} /{ }^{128} \mathrm{Xe}$ ratios of the samples after corrections for lowtemperature alteration and trapped component were converted to I-Xe ages by comparing with ${ }^{129} \mathrm{Xe} /{ }^{128} \mathrm{Xe}$ ratio of the Shallowater meteorite standard $(4.5633 \pm 0.0004$ Ga [1] irradiated with neutrons together with the samples.

The Zag meteorite is composed of light- and dark-colored portions, and the light portion shows older I-Xe ages and less SW noble gases than the dark portion [2]. This implies that the onset of the SW irradiation would have been between the I-Xe ages of light and dark portions. Our new I-Xe ages are associated with large errors, but the light portion was systematically older $(4.558-4.561 \mathrm{Ga})$ than the dark portion (4.551-4.554 Ga) and less enriched in SW noble gases, which are consistent with [2]. The weighted mean of I-Xe ages of the light and dark portions are $4.557 \pm 0.004 \mathrm{Ga}$ and $4.550 \pm$ $0.002 \mathrm{Ga}$, respectively, suggesting that the disk gas dissipation would have occurred $\sim 10 \mathrm{Myr}$ after the Solar System formation. The NWA 801 meteorite had younger IXe age $(4.549 \pm 0.012 \mathrm{Ga})$ and large amount of SW noble gases, which is similar to the Zag meteorite. Due to the large uncertainty in the I-Xe ages, more data with higher precision are required to tightly constrain the timing of the disk gas dissipation.

[1] Gilmour et al., (2006) Meteorit. Planet. Sci. 41, 19-31. [2] Bajo (2010) PhD thesis, Univ. Tokyo. 